\title{
ON THE CAPACITY OF WIRELESS SENSOR NETWORKS WITH OMNIDIRECTIONAL ANTENNAS
}

\author{
Onur Arpacioglu and Zygmunt J. Haas \\ School of Electrical and Computer Engineering \\ Wireless Networks Laboratory \\ Cornell University, Ithaca, NY 14853, USA \\ o.arpacioglu@cornell.edu, haas@ece.cornell.edu
}

\begin{abstract}
We establish some new results on the capacity of wireless sensor networks that employ single-userdetection, and we present the implications of our results on the scalability of such networks. In particular, we find bounds on the maximum achievable per-sensor end-to-end throughput, $\lambda_{e}$, and the maximum number of simultaneously successful wireless transmissions, $N_{t}^{\max }$, under a more general network scenario than previously considered. Furthermore, in the derivation of our results, we make no restrictions on the mobility pattern of the sensor and the destination nodes or on the number simultaneous transmissions and/or receptions that the nodes are capable of maintaining. In our derivation, we also analyze the effect of parameters such as the area of the network domain, $A$, the path loss exponent, $\gamma$, the processing gain, $G$, and the SINR threshold, $\beta$. Specifically, we prove the following results for a wireless sensor network of $N$ sensor nodes and $M$ destination nodes that are equipped with omnidirectional antennas:
\end{abstract}

(1) $\lambda_{c}$ is $\Theta(1 / N)$ under very general conditions that we identify in this paper.

(2) $N_{r}^{\max }$ has an upper bound that does not depend on $N$, which is the simultaneous transmission capacity of the network domain, $N_{t}^{Q}$. For a circular network domain, $N_{t}^{Q}$ is $O\left(A^{\min (\gamma / 2.11)}\right)$ if $\gamma \neq 2$ and $O(A / \log (A))$ if $\gamma=2$. In addition, $N_{t}^{Q}$ is $O\left(\gamma^{2}\right)$ and $O(G / \beta)$. Moreover, lack of attenuation and lack of space are equivalent, where $N_{t}^{Q}$ cannot exceed $1+G / \beta$.

(3) As $N \square \infty$ a desired per-sensor end-to-end throughput is not achievable, unless the average number of hops between a sensor-destination node pair does not grow indefinitely with $N$, both $M$ and $A$ grow with $N$ such that $M$ is $\Omega(N)$, and $N$ is $O\left(A^{\min \{\gamma / 2,1]}\right)$ if $\gamma \neq 2$ and $O(A / \log (A))$ if $\gamma=2$.

Keywords: Wireless sensor networks, capacity, scalability, throughput, omnidirectional transmission

This work has been supported in part by the DoD Multidisciplinary University Research Initiative (MURI) program administered by the Office of Naval Research under the grant number N00014-00-1-0564, the DoD Multidisciplinary University Research Initiative (MURI) program administered by the Air Force Office of Scientific Research under the grant number F49620-02-1-0233, and by the National Science Foundation grant number ANI-0081357.

This article is substantially based on our previous publication "On the Scalability and Capacity of Planar Wireless Networks with Omnidirectional Antennas" by $\mathrm{O}$. Arpacioglu and Z. J. Haas, published in the Wireless Communication and Mobile Computing Journal, vol. 4, issue 3. pp: 263-279 in May 2004. Copyright (C 2004 John Wiley \& Sons Ltd. Reproduced with permission.

\section{INTRODUCTION}

In recent years, there has been an increasing interest in certain class of wireless networks called wireless sensor networks. Typically, wireless sensor networks are deployed to collect information about an environmental variable by observing the information gathered at a set of destination nodes from a set of sensor nodes in the network. ${ }^{.}$

Scalability of wireless sensor networks has been an important research topic in the recent years, because of the growing demand to support a large number of nodes in future sensor networks, which are envisioned to consist of thousands to millions of sensor nodes. Two important questions in this context are: (1) Are wireless sensor networks scalable? In other words, is it possible to support a large number of nodes in a wireless sensor network? (2) If there are scalable patterns of wireless sensor networks, what are the conditions that govern their scalability?

To answer these questions, one must first define what scalability is. In this work, we interpret scalability as: the ability of the network to operate with a non-vanishing per sensor end-to-end throughput, bounded end-to-end delay, bounded power consumption, bounded processing power and bounded memory at each node, as the number of nodes grows large.

In this paper, we focus on the throughput aspect of scalability. Our objectives are: (1) to obtain theoretical results that show the dependencies among the per-sensor end-to-end throughput capacity $\lambda_{e}$, the number of sensor nodes $N$, and other parameters of a wireless sensor network, and (2) to determine the implications of these results on the scalability of such networks.

One of the most well known studies that stimulated the research on the capacity of wireless sensor networks was published by Gupta and Kumar [1]. In that paper, two network models were proposed to analyze the capacity of wireless networks. The first network model, the arbitrary network model, assumes that all $N$ nodes in the network are static, there are no restrictions on nodes' locations, and the network domain (i.e., the region within which the nodes are located) is a circular disk of area $1 \mathrm{~m}^{2}$. Each node is capable of maintaining at most one transmission or one reception at any given time. There are no restrictions on the choice of transmission powers, traffic pattern, routing protocol, and spatial-temporal transmission scheduling policy. The second model is the random network model, which assumes

\footnotetext{
${ }^{1}$ In the literature, sensor nodes are also called sources, and destination nodes are also called sinks, collectors or observers.
} 
a uniform distribution of node locations, a random traffic pattern, and a common transmission power that decreases with $N$, while ensuring the connectivity of the network as $N$ tends to infinity.

From a sensor network perspective, the arbitrary and the random network models correspond to sensor networks for which each node has the ability to be a sensor node and a destination node.

Additionally, in [1], two models for successful reception are proposed. The first reception model is the protocol model, which considers a transmission unsuccessful if the receiver is within the interfering range of an unintended transmitter. The second model is the physical model, which better represents realistic reception in practical wireless networks. In the physical model, for a transmission to be successful, the Signal-to-Interference-and-Noise Ratio, $S I N R$, at the receiver of the transmission has to be above some threshold value. It is assumed that the antennas are omnidirectional and that $P / x^{\gamma}$ is the power received at a distance $x$ from a given transmitter, where $P$ is the transmitted power and the path loss exponent $\gamma$ is assumed to be larger than 2 .

[1] concluded that, with the protocol model, $\lambda_{c}$ is ${ }^{2}$ $\Theta(1 / \sqrt{N})$ for arbitrary networks, whereas $\lambda_{e}$ is $\Theta(1 / \sqrt{N \log (\bar{N})})$ for random networks. With the physical model, they concluded that $\lambda_{e}$ is $O(1 / \sqrt[\gamma]{N})$ and $\Omega(1 / \sqrt{N})$ for arbitrary networks, whereas, $\lambda_{e}$ is $O(1 / \sqrt{N})$ and $\Omega(1 / \sqrt{N \log (N)})$ for random networks.

One of the assumptions of [1] is that all nodes are immobile. In [2], Grossglauser and Tse explored whether or not introducing mobility can increase $\lambda_{e}$. Their network model introduced some additional restrictions on the random network model of [1]. Firstly, they used the physical model, but allowed wideband communication by incorporating the processing gain, as to reduce interference. Secondly, the locations of the mobile nodes form a stationary ergodic process with a uniform stationary distribution in the network domain. Thirdly, as in [1], source-destination pairs do not change over time. Finally, they assumed that very long end-to-end packet delays are tolerable. Grossglauser and Tse concluded in [2] that there exists a routing and scheduling policy that delivers a packet to its destination with no more than two hops, and allows $\lambda_{e}$ to be $\Theta(1)$ as $N$ becomes large. Moreover, both [1] and [2] concluded that it is possible to schedule $\Theta(N)$ many simultaneously successful transmissions in a wireless network.

In [3], Marco et al. explored $\lambda_{e}$ with two particular traffic patterns. They used a half-duplex reception model, which is based on the protocol model, and they assumed that the $N$ sensor nodes are static, they are uniformly distributed in the circular network domain, and the transmission range is

\footnotetext{
2 We will use the standard asymptotic notations: Let $f$ and $g$ be nonnegative functions of a variable $x . f$ is $O(g)$ with respect to $x$, if there exist positive real numbers $x_{0}$ and $y_{0}$, such that $0 \leq f \leq y_{0} g$ for every $x \geq x_{0} . f$

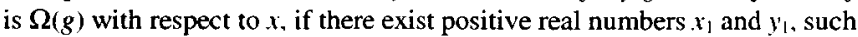
that

$0 \leq y_{1} g \leq f$ for every $x \geq x_{1} . f$ is $\Theta(g)$ with respect to $x$, if $f$ is both $O(g)$ and $\Omega(g)$ with respect to $x$. We will omit the phrase "with respect to $x$ " when it can be understood from the context. Also, we will make use of the fact that $f$ is $\Theta(g)$ with respect to $x$ if $f(x) / g(x)$ has limit in $(0, \infty)$ as $x \rightarrow \infty$.
}

constant. The first traffic pattern that they considered is the all-to-one model, where each sensor node has the same destination node which is located at the center of the network domain at all times. The second traffic pattern is the all-to-all model, where each sensor node has to broadcast the information that it generates to all other sensor nodes in the network. For these two traffic patterns, they concluded that $\lambda_{e}$ is $\Theta(1 / N)$.

In [4], Chakrabarti et al. concluded that $\lambda_{c}$ is $\Theta(\log (N) / N)$ for the random network model of [1], if the all-to-one traffic pattern and an adaptive transmission rate model are used instead of the random traffic pattern and the threshold SINR based reception model, respectively.

In [5], Toumpis and Goldsmith also used an adaptive rate model, and using numerical methods, they evaluated the effect of spatial reuse, multi-hop routing and power control for a particular placement of the nodes. They concluded that each of these schemes provides expansions of the capacity region that is defined by the set of achievable rates between the nodes.

In [6], $\mathrm{Li}$ et al. pointed out the effect of traffic pattern on $\lambda_{r}$ and concluded that only wireless networks with local traffic patterns can be scalable.

Usage of directional antennas at the transmitters or the receivers can provide significant increases in $\lambda_{c}$, depending on how narrow the width of the antenna's main lobe and how small the side lobes of the antenna radiation pattern can be made. For example, in [7], using a sender-based interference model, Yi, Pei, and Kalyanaraman investigated the improvement in $\lambda_{e}$ provided by the usage of directional antennas for arbitrary and random wireless networks.

The ability of a node to maintain multiple simultaneous transmissions and/or receptions can also provide an increase in $\lambda_{e}$. For example, in [8], using directional antennas, Peraki and Servetto studied random networks with and without multiple simultaneous transmission or reception capability, and they concluded that an improvement of at most $\Theta\left(\log ^{2}(N)\right)$ can be achieved over the $\Theta(1 / \sqrt{N \log (N)})$ result of [1].

Deployment of a wired backbone can also provide an increase in $\lambda_{e}$, since it allows reducing average number of wireless transmissions per packet. For example, in [9], Liu et al. considered the benefit of deploying base stations connected to a wired backbone in the random network of [1]. They concluded that if the number of base stations grows asymptotically faster than $\sqrt{N}$, then aggregate throughput capacity increases linearly with the number of base stations.

The results of studies such as [1]-[9] are based on the assumption that the nodes are equipped with single-userdetection based receivers, which are widely used in today's practical wireless networks. These types of receivers attempt to detect a particular transmission, while treating all other interfering transmissions as noise. On the other hand, studies such as [10]-[12] concluded that sizable gains in network throughput can be provided by not treating interference simply as noise, but rather employing more sophisticated receivers and implementing certain cooperation strategies among the nodes during encoding, transmission and decoding stages of each communication. 
Our work has been motivated by the desire to relax some of the limitations of [1]-[4], and to improve on their models. In particular, the radio propagation model of [1], [2] and [4], (i.e., $P / x^{\gamma}$ ) becomes inappropriate as the transmitterreceiver separation becomes small. Since the network domain in these studies has a fixed area, as $N$ grows large, the intended transmitter-receiver pairs get arbitrarily close to each other, which leads to a very optimistic evaluation of the SINR values due to the assumed propagation model. To avoid this problem, we use a bounded propagation model, which we discuss in Section 2.3. In terms of mobility, in [1], [3] and [4] the nodes are immobile, while in [2] the mobility pattern is a very special one. In our work, we allow for a general mobility pattern of the nodes. Furthermore, by allowing a general traffic pattern, we relax the assumption of [1]-[4] which states that the source-destination pairs never change over time, and the assumption of [3] and [4], which states that the traffic pattern is either all-to-one or allto-all. Moreover, in [1]-[4] each node can maintain either a single transmission or reception at any given time, whereas our work also considers the situtation when the nodes have the ability to maintain multiple transmissions and/or receptions at the same time. In addition, we also analyze the effect of parameters such as $A, \gamma, G$ and $\beta$ on $\lambda_{e}$, none of which has been addressed in previous works. Above all, we evaluate the implications of our results on the scalability of wireless sensor networks.

The main contribution of this study is in the derivation of new results on the capacity and scalability of wireless sensor networks through the use of a more general network model and a more realistic bounded propagation model, as compared with the models of the previous studies.

The rest of the paper is organized as follows: Section 2 presents our network model, and Section 3 provides related definitions. In Section 4, we derive the upper bounds on the simultaneous transmission capacity and the per-sensor endto-end throughput capacity. In Section 5, we analyze the derived upper bounds, together with illustrative figures. Section 6 establishes the tightness of the scaling behavior of $\lambda_{c}$ with respect to $N$. Section 7 discusses the implications of our results on scalability. Finally, we conclude in section 8 .

\section{SENSOR NETWORK MODEL}

In this section, we explain the assumptions underlying our results. Some of these assumptions are quite general, allowing our upper bound results to hold even in wireless sensor networks that are configured with optimal settings of the parameters. Although this paper is primarily targeted at the sensor networks, the generality of our assumptions allows straightforward extensions of the results of this paper to many other types of wireless networks, such as wireless ad hoc networks. We will elaborate on some other consequences of these general assumptions after presenting our results.

\section{3.network domain and nodes}

We denote the total number of nodes in the network by $N_{\text {toral }}$. There are two types of nodes in the network: sensor nodes and destination nodes. There are $N$ sensor nodes and
$M$ destination nodes. Any given node is either a sensor node or a destination node or both of them, and the type of the node remains the same at all times. Two immediate consequences of these assumptions are that $N_{\text {total }} \leq N+M$, and that $N_{\text {total }}=N+M$ if and only if the set of sensor nodes and the set of destination nodes are disjoint.

The task of each sensor node is to send the collected information to a destination node (of course, to a destination node other than itself, if the node itself happens to be a destination node, too). We assume that $M \leq N$. Typically, this inequality is satisfied with "«" in practical wireless sensor networks. An immediate consequence of the inequality $M \leq N$ is that $N_{\text {total }}=N$ if and only if all destination nodes are also sensor nodes. Note that this case corresponds to the network models of [1] and [2].

Network domain is defined to be the space within which each node is constrained to reside. We denote this space by $\boldsymbol{Q}$. We will assume that $\boldsymbol{Q}$ is a closed disk with a diameter $D$ and an area $A$. There are no restrictions on the mobility pattern of the nodes within $Q$.

\section{4.transmitter and receiver model}

Each of the nodes is capable of being a transmitter and/or a receiver at any given time. All transmitters and receivers are equipped with omnidirectional antennas. There are no restrictions on the variation of transmission power during a transmission or on the number of simultaneous transmissions and/or receptions that a node is capable of maintaining. Hence, the assumption in [1]-[4] that a node is capable of maintaining either one transmission or one reception at any given time is one of the many cases covered by our model. For the time being, we assume that all transmissions take place within the same communication bandwidth, but we will relax this assumption later. At the intended receiver of a transmitted signal, all of the remaining received signals are considered as interference. $\zeta_{i}(t)$ is the power of the thermal noise present in the communication bandwidth at receiver $i$ at time $t$. We assume that each of the receivers can receive information intended for itself reliably at a rate not larger than $W_{\max }$ bits/s and only when the SINR at the receiver is greater than or equal to the SINR threshold, $\beta$. Information received when the above condition does not hold is considered unreliable and, thus, discarded. In this work, we let $\beta$ be any positive real number. In general, $\beta$ is dependent on the modulation scheme, the required bit error rate of the received information, the required transmission rate, and the type of the error control code. The processing gain, $G$, is the factor by which the total received interference power is reduced at each of the receivers. In this work, we let $G$ be any positive real number. Typically $G>1$ for wideband communication systems, such as spread spectrum CDMA, and is taken to be 1 for narrowband communications.

\section{5.propagation model}

For any given two given transmissions, let $i$ and $j$ be the indices that represent the transmissions. Let $P_{i}^{j}(t)$ be the 
power transmitted by transmitter $j$ at time $t$. Let $d_{j i}(t)$ be the distance $e^{3}$ between transmitter $j$ and receiver $i$ at time $t$. Let $P_{r}^{\prime i}(t)$ denote the power received by receiver $i$ from transmitter $j$ at time $t$. We will assume that

$$
P_{r}^{j i}(t)=P_{i}^{j}(t) a\left(d_{j i}(t)\right) \text {, }
$$

where $a(x)$ is the attenuation function. One of the most commonly used expressions for $a(x)$ is $1 / x^{\gamma}$, where $\gamma \geq 0$ is the path loss exponent. ${ }^{4}$ However, this expression becomes inappropriate as $x$ becomes smaller than 1 , as it results in receiving power that is larger than the transmitted power. In fact, the received power in the formula approaches infinity as $x$ tends to zero. Of course, this is unrealistic and is an artifact of the inappropriateness of the expression for small distances. ${ }^{5}$ The inappropriateness of the $1 / x^{\gamma}$ formula was also noticed in some previous works on connectivity, and to obtain more meaningful results at small distances, while approximating the conventional model at large distances, the following alternative propagation model was proposed in those studies (see, e.g., [15] and [16]):

$$
a(x)=\frac{1}{(1+x)^{\gamma}}, \quad x \geq 0 .
$$

We call this propagation model, the power law decaying propagation model, ${ }^{6}$ and we will use this model in our calculations.

\section{traffic pattern}

We make no restrictions on the temporal variation of the destination of each of the sensor nodes, the selection of the intermediate nodes that are involved in routing the information originated by the sensor nodes, and the segmentation of information, so that different segments can possibly be transmitted over different paths and at different times. Note that, together with the assumptions that we stated in Section 2.1, these imply that the class of traffic

In this paper, all distance measures and area measures are in units of "meter" and "meter"." respectively.

${ }^{+}$In free space $\gamma=2$, but in realistic mobile radio channels, $\gamma$ can take values between 1.6 and 6 (see [13] and [14]).

The precise reason for this problem can be explained as follows: Consider the free space case. In the derivation of the received power expression. a unity gain point source in free space is assumed and the flux of the transmitted power $P_{l}$, per unit surface area of the sphere with radius $x$ around the source is calculated. The resulting power flux density expression, $P_{t} / 4 \pi x^{2}$, has the unit Watts/meter. The wave-front of the transmission occupies only part of the aperture of the receiving antenna, so that the power captured by the aperture results from only that part of the wave-front that is seen by the aperture. To quantify this partial aperture area occupied by the wave-front, effective aperture area, $A_{e}$, is defined as the ratio of the available power at the terminals of the receiver antenna to the power flux density at the location of the receiver antenna. In general, $A_{e}$ depends on the physical characteristics of the receiver antenna and the distance $x$ between the transmitter and the receiver antennas. In [1]. [2] and [4], $A_{c}$ is assumed to be independent of $x$ and is taken to be $4 \pi$ meter $^{2}$, so that the received power expression simplifies to $P_{t} / x^{2}$. In fact, as $x \rightarrow 0$, the power flux density approaches infinity, and with the constant $A_{e}$ assumption, the received power also approaches infinity. This shows that $A_{c}$ should not be taken as a constant for small values of $x$. In fact, $A_{e}$ should approach zero as $x$ approaches zero, so that the received power, $A_{c} P_{t} / 4 \pi x^{2}$, never exceeds $P_{t}$.

- The corresponding $A_{c}$ for this model in free space is equal to $4 \pi r^{2} /(1+x)^{2}$ meter ${ }^{2}$. Note that this expression converges to $4 \pi$ meter $^{2}$ as $x$ becomes large. which is also the assumed aperture area in the conventional model. patterns that our model covers is indeed a very large class: in particular it contains all many-to-many type traffic patterns for which the set of sensor nodes is at least as large as the set of destination nodes. Hence, this class contains the two specific traffic patterns considered in [3], as well.

On the other hand, as in [1]-[4], we assume that intermediate nodes that act as relays do not jointly encode and transmit chunks of information that are gathered from distinct transmissions.

Finally, we denote the average number of hops between the originating sensor node and the destination of $a$ bit by $\bar{H}$, and we let it be any real number larger than or equal to 1 , since each bit has to be transmitted over at least one hop to reach its destination.

\section{DEFINITIONS}

We define a transmission at an arbitrary time $t$ to be a successful transmission, if the SINR at the intended receiver of the transmission at time $t$ is not smaller than $\beta$. We denote the number of simultaneously successful transmissions at time $t$ by $N_{t}$. We define the simultaneous transmission capacity of the sensor network, $N_{t}^{\max }$, as the maximum value of $N_{t}$ over all the placements of the nodes, the choices of the transmitters, their intended receivers, and the transmission powers. Next, we define the simultaneous transmission capacity of the network domain, $N_{t}^{Q}$ as the maximum value of $N_{t}$ over all the placements of the nodes, the choices of the transmitters, their intended receivers, and the transmission powers, given that there are no restrictions on the number of nodes in the network. An immediate consequence of these definitions is that $N_{t}^{\max } \leq N_{t}^{Q}$.

Let $b_{i}(T)$ be the total amount of bits of information generated by sensor node $i$ and received by its destinations during a $T$ second time interval $[0, T]$. We define the end-toend throughput of sensor node $i, \lambda_{i}$, as follows:

$$
\lambda_{i}:=\lim _{T \rightarrow \infty} \frac{b_{i}(T)}{T}, \quad 1 \leq i \leq N .7
$$

We also define the per-sensor average end-to-end throughput as the arithmetic mean of $\lambda_{i}$ 's, i.e.,

$$
\lambda:=\frac{1}{N} \sum_{i=1}^{N} \lambda_{i}
$$

Next, we propose two achievability definitions: an endto-end throughput $\lambda_{0}$ is said to be achievable by all sensor nodes, if there exist a mobility pattern of the nodes, a traffic pattern, a spatial-temporal transmission scheduling policy, and a temporal variation of transmission powers, so that $\lambda_{i} \geq \lambda_{0}$ for all $1 \leq i \leq N$. Likewise, an end-to-end throughput $\lambda_{0}$ is said to be achievable on average, if there exist a mobility pattern of the nodes, a traffic pattern, a spatial-temporal transmission scheduling policy, and a temporal variation of transmission powers so that $\lambda \geq \lambda_{0}$. Note that if $\lambda_{0}$ is achievable by all sensor nodes, then it is also achievable on average, and if $\lambda_{0}$ is not achievable on average, then it is not achievable by all sensor nodes, either. Hence, we will

${ }^{7}$ Our results also apply with a more general definition of throughput, where "lim" is replaced with "liminf". 
shortly say that $\lambda_{0}$ is not achievable if $\lambda_{0}$ is not achievable on average.

Finally, we propose two capacity definitions: the persensor end-to-end throughput capacity, $\lambda_{e}$, is the supremum of all end-to-end throughputs that are achievable by all sensor nodes. The per-sensor average end-to-end throughput capacity, $\lambda_{m}$, is the supremum of all end-to-end throughputs that are achievable on average. An immediate consequence of these definitions is that $\lambda_{m} \geq \lambda_{e}$.

\section{DERIVATION OF THE UPPER BOUNDS}

\section{upper boundS on simultaneous transmission capacity}

In this subsection, we prove the following theorem that provides upper bounds on $N_{t}^{\max }$ and $N_{t}^{Q}$ :

Theorem 1: Let $t$ be an arbitrary time instant. Then,

(i) the simultaneous transmission capacities of the sensor network and the network domain have the following upper bounds:

$$
\begin{aligned}
& N_{t}^{\max } \leq N_{l}^{Q} \leq U_{\gamma}, \\
& N_{t}^{\max } \leq N_{\text {total }}(1+G / \beta),
\end{aligned}
$$

where

$$
U_{\gamma}:= \begin{cases}\frac{(\gamma-1)(\gamma-2)\left(1+\frac{G}{\beta}\right) d^{2}}{2\left(1+\frac{\gamma^{2}}{(1+d)^{\gamma-1}}-\frac{\gamma-1}{(1+d)^{\gamma-2}}\right)} & \gamma \notin\{1,2\} \\ \frac{\left(1+\frac{G}{\beta}\right) d}{2\left(1-\frac{\log (1+d)}{d}\right)} & \gamma=1, \\ \frac{\left(1+\frac{G}{\beta}\right) d^{2}}{2\left(\log (1+d)-\frac{d}{1+d}\right)} & \gamma=2, \\ d:=D / \sqrt{\frac{2}{3}-\frac{\sqrt{3}}{2 \pi}} . & \text { (T1.6) }\end{cases}
$$

(ii) no node can be receiving more than $1+G / \beta$ successful transmissions at time $t$.

\section{Proof of Theorem 1:}

Recall that $N_{t}$ is the number of simultaneously successful transmissions at time $t$. Now, index each transmitter-receiver pair that belongs to the same transmission with a unique number between 1 and $N_{t}$. So, receiver $i$ is the intended receiver of transmitter $i$ for every $1 \leq i \leq N_{t}$. Let $\operatorname{SINR}_{i}(t)$ be the SINR at receiver $i$ at time $t$. Then

$$
\operatorname{SINR}_{i}(t)=\frac{P_{r}^{i i}(t)}{\zeta_{i}(t)+\frac{1}{G} \sum_{\substack{j=1 \\ j \neq i)}}^{N_{t}} P_{r}^{\prime \prime}(t)}, \quad 1 \leq i \leq N_{t} .
$$

(1)

From the definition of a successful transmission, $N_{t}$ simultaneously successful transmissions can take place at time $t$ if and only if

$$
\begin{aligned}
& \operatorname{SINR}_{i}(t) \geq \beta, \\
& 1 \leq i \leq N_{r}, \\
& \Rightarrow \frac{1}{G} \sum_{\substack{j=1 \\
(j \neq i)}}^{N_{t}} P_{r}^{j i}(t) \leq \frac{P_{r}^{i i}(t)}{\beta}-\zeta_{i}(t), \\
& 1 \leq i \leq N_{r}
\end{aligned}
$$

$$
\begin{array}{ll}
\stackrel{(a)}{\Leftrightarrow} \frac{1}{G} \sum_{\substack{j=1 \\
j \neq i}}^{N_{t}} P_{t}^{j}(t) a\left(d_{j i}(t)\right) \leq \frac{P_{r}^{i i}(t)}{\beta}-\zeta_{i}(t), & 1 \leq i \leq N_{t}, \\
\stackrel{(b)}{\Leftrightarrow} \frac{1}{G} \sum_{\substack{j=1 \\
j \neq i}}^{N_{t}} \frac{P_{t}^{j}(t) a\left(d_{j i}(t)\right)}{P_{i}^{i}(t) a\left(d_{i i}(t)\right)} \leq \frac{1}{\beta}-\frac{\zeta_{i}(t)}{P_{r}^{i}(t) a\left(d_{i i}(t)\right)}, \quad 1 \leq i \leq N_{t},
\end{array}
$$

where step ( $a$ ) follows from (a.1), and step ( $b$ ) follows from dividing both sides by $P_{r}^{i i}(t)=P_{r}^{i}(t) a\left(d_{i i}(t)\right)$.

In general, for $0 \leq x \leq y+z, y \geq 0$, and $z \geq 0$,

$$
\frac{1}{(1+x)^{\gamma}} \geq \frac{1}{(1+y+z)^{\gamma}} \geq \frac{1}{(1+y+z+y z)^{\gamma}}=\frac{1}{(1+y)^{\gamma}(1+z)^{\gamma}} \text {. }
$$

Therefore, for $a(x)$ as defined by (a.2),

$$
a(x) \geq a(y) a(z), 0 \leq x \leq y+z, y \geq 0, \text { and } z \geq 0 .
$$

(3)

Now, let $l_{j i}(t)$ be the distance between receiver $j$ and receiver $i$ at time $t$. Then, from the triangle inequality,

$$
d_{j i}(t) \leq d_{j j}(t)+l_{j i}(t), \quad 1 \leq i \leq N_{t} \text { and } 1 \leq j \leq N_{t} .
$$

Setting $x=d_{j i}(t), y=d_{j j}(t)$ and $z=l_{j i}(t)$ in (3) we find that

$$
a\left(d_{j i}(t)\right) \geq a\left(d_{j j}(t)\right) a\left(l_{j i}(t)\right) .
$$

Thus, from (2) and (4), if $N_{t}$ simultaneously successful (T1. transmissions can take place at time $t$, then

$$
\sum_{\substack{j=1 \\ j \neq i}}^{N_{i}} \frac{P_{t}^{j}(t) a\left(d_{j j}(t)\right) a\left(l_{j i}(t)\right)}{P_{i}^{i}(t) a\left(d_{i i}(t)\right)} \leq \frac{G}{\beta}-\frac{G \zeta_{i}(t)}{P_{i}^{i}(t) a\left(d_{i i}(t)\right)}, \quad 1 \leq i \leq N_{,} .
$$

Define,

(6)

$$
p_{j i}(t):=\frac{P_{t}^{i}(t) a\left(d_{j j}(t)\right)}{P_{i}^{i}(t) a\left(d_{i i}(t)\right)}
$$

Next, we add all inequalities in (5), while incorporating (6). Hence, if $N_{t}$ simultaneously successful transmissions can take place at time $t$, then

$$
\begin{aligned}
& \sum_{i=1}^{N_{t}} \sum_{\substack{j=1 \\
j \neq i}}^{N_{t}} a\left(l_{j i}(t)\right) p_{j i}(t) \leq \frac{G N_{t}}{\beta}-\sum_{i=1}^{N_{t}} \frac{G \zeta_{i}(t)}{P_{t}^{i}(t) a\left(d_{i i}(t)\right)} \\
\Rightarrow & \sum_{i=1}^{N_{t}-1} \sum_{j=i+1}^{N_{t}}\left(a\left(l_{j i}(t)\right) p_{j i}(t)+a\left(l_{i j}(t)\right) p_{i j}(t)\right) \leq \frac{G N_{t}}{\beta} \\
(a) & \sum_{i=1}^{N_{t}-1} \sum_{j=i+1}^{N_{t}} a\left(l_{i j}(t)\right)\left(p_{j i}(t)+p_{i j}(t)\right) \leq \frac{G N_{t}}{\beta} \\
(b) & \sum_{i=1}^{N_{t}-1} \sum_{j=i+1}^{N_{t}} 2 a\left(l_{i j}(t)\right) \leq \frac{G N_{t}}{\beta} \\
(c) & \sum_{i=1}^{N_{t}-1} \sum_{\substack{j=i+1 \\
N_{t}}}^{N_{t}}\left(a\left(l_{j i}(t)\right)+a\left(l_{i j}(t)\right)\right) \leq \frac{G N_{t}}{\beta} \\
\Leftrightarrow & \sum_{i=1}^{N_{t}} \sum_{\substack{j=1 \\
(j \neq i}}^{N_{t}} a\left(l_{j i}(t)\right) \leq \frac{G N_{t}}{\beta} \\
\text { (d) } & \Leftrightarrow \sum_{i=1}^{N_{t}} \sum_{\substack{j=1 \\
j \neq i}}^{N_{t}} a\left(l_{i j}(t)\right) \leq \frac{G N_{t}}{\beta},
\end{aligned}
$$


where steps $(a),(c)$, and $(d)$ follow from the fact that $a\left(l_{j i}(t)\right)=$ $a\left(l_{i j}(t)\right)$ for every $i, j, t$, and step $(b)$ follows from the fact that $x+1 / x \geq 2$ for every positive real number $x$.

From (7), we observe that the problem of obtaining an upper bound on $N_{t}$ can be reduced to finding a lower bound on the summation term on the left-hand side of inequality (7). This term involves the sum of the attenuation function evaluated at the inter-receiver distances defined by the $N,\left(N_{t}-1\right) / 2$ pairs of receivers. To find the lower bound, we make use of Lemma 1 , which is derived in the next subsection.

\section{interpoint distance sum inequality}

In this subsection, we derive a lemma that gives an upper bound on the sum of the square of the nearest, the second nearest, $\ldots$, and the $(n-1)^{s t}$ nearest neighbor distances from each of the $n$ points that are arbitrarily located in a disk of diameter $D$.

Lemma 1: (Interpoint distance sum inequality for a disk) Let $B(D)$ be a disk having diameter $D$. Let $n$ points be arbitrarily placed in $B(D)$. Suppose each point is indexed by a distinct integer between 1 and $n$. Let $l_{i j}$ be the Euclidean distance between point $i$ and point $j$. Define the $m^{\text {th }}$ closest point to point $i, a_{i m}$, and the Euclidean distance between point $i$ and the $m^{\text {th }}$ closest point to point $i, u_{i m}$, as follows:

$$
\begin{aligned}
& a_{i 1}:=\underset{\substack{j \in\{1,2, \ldots, n\} . \\
j \neq i}}{\arg \min }\left\{l_{i j}\right\}, \quad 1 \leq i \leq n, \\
& a_{i m}:=\underset{\substack{j \in\{1,2, \ldots n\} \\
j \in\{i\} \cup\left\{a_{1}, w^{m-1}\right.}}{\arg } \min _{i j}\left\{l_{i j}\right\}, \quad 1 \leq i \leq n \text { and } 2 \leq m \leq n-1 \text {, } \\
& u_{i m}:=l_{i a_{t u}}, \quad \quad 1 \leq i \leq n \text { and } 1 \leq m \leq n-1 .
\end{aligned}
$$

Then

$$
\sum_{i=1}^{n} u_{i m}{ }^{2} \leq \frac{m D^{2}}{c_{2}}, \quad 1 \leq m \leq n-1,
$$

(L1.1)

where $c_{2}:=\frac{2}{3}-\frac{\sqrt{3}}{2 \pi}$.

\section{Proof of Lemma 1:}

The proof involves a spherical geometric approach, which is used to solve similar problems in [17]. Let $B_{i}(x)$ denote the disk of diameter $x$, whose center is at point $i$. Consider the following sets of disks:

$$
R_{m}:=\left\{B_{i}\left(u_{i m}\right): 1 \leq i \leq n\right\}, \quad 1 \leq m \leq n-1 .
$$

Let us first consider the disks in $R_{1}$. All disks in $R_{1}$ are nonoverlapping. ${ }^{8}$ This can be proven by contradiction: Suppose that there exist two points $i$ and $j$ such that $B_{i}\left(u_{i 1}\right)$ and $B_{j}\left(u_{j 1}\right)$ are overlapping. This, by the definition of overlapping, implies $\left(u_{i 1}+u_{j 1}\right) / 2>l_{i j}$. Without loss of essential generality, suppose $u_{i 1} \geq u_{j 1}$. Then $u_{i 1}>l_{i j}$. However, this would contradict the definition of $u_{i 1}$. Therefore, our original assumption, i.e., the existence of two overlapping disks in $R_{1}$, is invalid.

Let $A(X)$ denote the area of a region $X$. If $X$ is a disk with diameter $a$, then $A(X)=\pi a^{2} / 4$. Next, we find a lower bound

\footnotetext{
${ }^{8}$ Two disks are defined to be overlapping if the distance between the centers of the disks is smaller than the sum of the radii of the two disks.
}

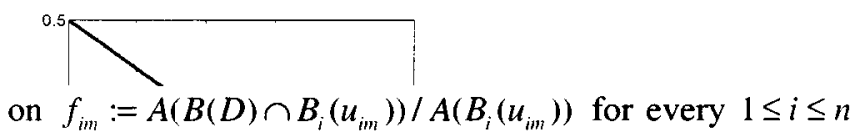
and $1 \leq m \leq n-1$. Pick any point $S$ from the periphery of $B(D)$ and consider the following overlap ratio:

$f_{i m}^{S}:=A\left(B(D) \cap B_{S}\left(u_{i m}\right)\right) / A\left(B_{S}\left(u_{i m}\right)\right), 1 \leq i \leq n, 1 \leq n \leq n-1$. Geometrical computation of $f_{i m}^{S}$ using Figure 1 leads to the following formula: $f_{i m}^{S}=\left.f(y)\right|_{y=\frac{u_{i m}}{D}}$, where

$$
f(y):=\frac{1}{\pi}\left(1-\frac{2}{y^{2}}\right) \arccos \left(\frac{y}{2}\right)+\frac{1}{y^{2}}-\frac{1}{\pi} \sqrt{\frac{1}{y^{2}}-\frac{1}{4}} .
$$

Figure 2 shows the variation of $f(y)$ with $y$. Since $f(y)$ is a decreasing function of $y$ and $u_{i m} \leq D, f_{i m}^{s} \geq f(1)$. Also, $f_{i m} \geq f_{i m}^{S}$. Hence, by defining $c_{2}:=f(1)$, we obtain the following lower bound on $f_{i m}$ for every $1 \leq i \leq n$ and $1 \leq m \leq n-1$ :

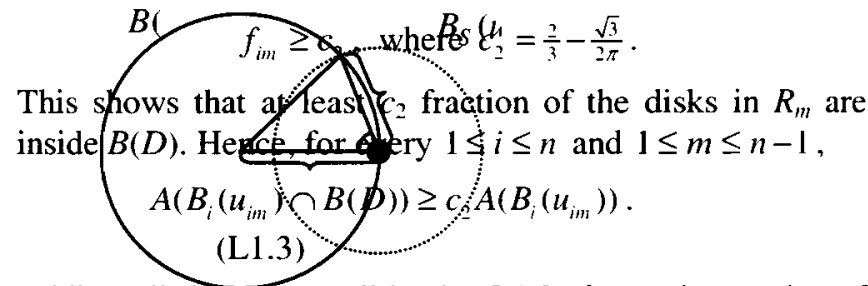

Adding all the $n$ inequalities in (LI.3) for a given value of $m$, we obtain the following inequality for every $1 \leq m \leq n-1$ :

$$
\sum_{i=1}^{n} A\left(B_{i}\left(u_{i m}\right) \cap B(D)\right) \geq c_{2} \sum_{i=1}^{n} A\left(B_{i}\left(u_{i m}\right)\right) .
$$

Since all disks in $R_{1}$ are non-overlapping,

$$
\sum_{i=1}^{n} A\left(B_{i}\left(u_{i 1}\right) \cap B(D)\right) \leq A(B(D))
$$

(L1.4) and (L1.5) imply,

$$
A(B(D)) \geq c_{2} \sum_{i=1}^{n} A\left(B_{i}\left(u_{i 1}\right)\right)
$$

In $\quad(\mathrm{L} 1.6), \quad A(B(D))=\pi D^{2} / 4 \quad$ and $\quad A\left(B_{i}\left(u_{i 1}\right)\right)=\pi u_{i 1}{ }^{2} / 4$. Substituting these equalities into (L1.6) and dividing both sides by $\pi c_{2} / 4$, we obtain,

$$
\sum_{i=1}^{n} u_{i 1}{ }^{2} \leq \frac{D^{2}}{c_{2}}
$$

Next, let us consider the disks in $R_{m}$ for every $2 \leq m \leq n-1$. In this case, there can be overlaps between some pairs of disks in $R_{m}$. Consider two overlapping disks, $B_{i}\left(u_{i m}\right)$ and $B_{j}\left(u_{j m}\right)$ centered at the points $i$ and $j$, respectively. Now we show that if $u_{i m} \geq u_{j m}$ then $j \in S_{i m}$, where $S_{i m}:=\left\{a_{i k}: 1 \leq k \leq\right.$ 
$m-1\}$. This can be proven by contradiction: Suppose $j \notin S_{i m}$ when $u_{i m} \geq u_{j m}$. Since $B_{i}\left(u_{i m}\right)$ and $B_{j}\left(u_{j m}\right)$ are overlapping, $\left(u_{i m}+u_{j m}\right) / 2>l_{i j}$. Then, since $u_{i m} \geq u_{j m}, u_{i m}>l_{i j}$. However, together with our assumption $j \notin S_{i m}$, this would contradict the definition of $u_{i m}$. Therefore, our original assumption, i.e., $j \notin S_{i m}$, is invalid.

Next, we show that any arbitrarily chosen point within $B(D)$ does not belong to more than $m$ overlapping disks from $R_{m}$. The proof is again by contradiction: Suppose there is a point in $B(D)$ that belongs to more than $m$ overlapping disks from $R_{m}$. Take the largest $m+1$ of these overlapping disks. Consider the largest disk. Let $b$ be the point at the center of this largest disk. Then all other $m$ disks belong to $S_{b m}$ due to the result proved in the previous paragraph. However, this contradicts the fact that the cardinality of $S_{b m}$ is $m-1$. Therefore, our original assumption, i.e., the existence of a point belonging to more than $m$ overlapping disks from $R_{m}$, is invalid.

Since any chosen point within $B(D)$ can belong to at most $m$ overlapping disks from $R_{m}$, then for every $2 \leq m \leq n-1$, we have

$$
\sum_{i=1}^{n} A\left(B_{i}\left(u_{i m}\right) \cap B(D)\right) \leq m A(B(D)) .
$$

(L1.8)

(L1.4) and (L1.8) imply,

$$
\sum_{i=1}^{n} u_{i m}^{2} \leq \frac{m D^{2}}{c_{2}}, 2 \leq m \leq n-1 .(\mathrm{Ll} 1.9)
$$

Combining (L1.7) and (L1.9) completes the proof.

$\square$

\section{application of the interpoint distance sum inequality}

In this subsection, we derive Lemma 2, which in combination with Lemma 1 , provides a necessary condition for $N_{t}$ simultaneously successful transmissions. Next, by using this necessary condition and another lemma, Lemma 3 , we complete the derivation of the upper bound on $N_{t}, N_{t}^{Q}$ and $N_{t}^{\max }$.

In Lemma 1 , by setting $n=N$, and the location of the points as the location of the receivers at time $t, u_{i m}(t)$ becomes the Euclidean distance between receiver $i$ and the $m^{\text {th }}$ closest receiver to receiver $i$ at time $t$. Hence, we obtain the following inequality:

(8)

$$
\sum_{i=1}^{N_{t}}\left[u_{i m}(t)\right]^{2} \leq \frac{m D^{2}}{c_{2}}, \quad 1 \leq m \leq N_{t}-1 .
$$

, from (7), we obtain the following necessary condition for $N_{t}$ simultaneously successful transmissions at time $t$ :

$$
\begin{aligned}
& \sum_{m=1}^{N_{t}-1} \sum_{i=1}^{N_{t}} a\left(u_{i m}(t)\right)=\sum_{i=1}^{N_{t}} \sum_{\substack{j=1 \\
j \neq i}}^{N_{t}} a\left(l_{i j}(t)\right) \leq \frac{G N_{t}}{\beta} \\
\Leftrightarrow & \sum_{m=1}^{N_{t}-1} \sum_{i=1}^{N_{t}} \frac{1}{\left(1+u_{i m}(t)\right)^{\gamma}} \leq \frac{G N_{t}}{\beta} .
\end{aligned}
$$

To incorporate the constraint of (8) into (9), we use the following lemma:

Lemma 2: For $n \geq 1$, let $x_{1}, x_{2}, \ldots, x_{n}, C$ be nonnegative real numbers satisfying the following inequality:

$$
\sum_{i=1}^{n} x_{i}^{2} \leq C
$$

Let $b$ be a nonnegative real number. Then

$$
\sum_{i=1}^{n} \frac{1}{\left(1+x_{i}\right)^{b}} \geq \frac{n}{\left(1+\sqrt{\frac{c}{n}}\right)^{b}} .
$$

Proof of Lemma 2:

For $b=0,(\mathrm{~L} 2.2)$ is satisfied with equality. Thus, we consider the case when $b>0$. Define the column vector $x$ and the multivariate function $f(x)$ as follows:

$$
\begin{aligned}
\boldsymbol{x} & :=\left[x_{1} x_{2} \ldots x_{n}\right]^{T}, \\
f(\boldsymbol{x}) & :=\sum_{i=1}^{n} \frac{1}{\left(1+x_{i}\right)^{b}} .
\end{aligned}
$$

We use Kuhn-Tucker Theory from [18] to find the minimum value of $f(x)$ subject to the constraint in (L2.1). We define the constraint function, $g(\boldsymbol{x})$ as follows:

$$
g(x):=C-\sum_{i=1}^{n} x_{i}^{2} \geq 0 \text {. }
$$

Let $\boldsymbol{y}=\left[\begin{array}{llll}y_{1} & y_{2} & \ldots . & y_{n}\end{array}\right]^{T}$ be the column vector at which $f$ takes its minimum value. Then, from Kuhn-Tucker Theory, there exists $\theta \geq 0$, such that the following conditions are satisfied:

$$
\left.(\nabla f(x)-\theta \nabla g(x))\right|_{x=y} \geq 0,
$$

$$
\begin{array}{r}
\left.\boldsymbol{y}^{T}(\nabla f(\boldsymbol{x})-\theta \nabla g(\boldsymbol{x}))\right|_{\boldsymbol{x}=\boldsymbol{y}}=0, \\
\theta g(\boldsymbol{y})=0,
\end{array}
$$

$$
g(y) \geq 0
$$

(L2.6)

From (L2.3) and (L2.4) we obtain,

$$
\begin{aligned}
& \frac{-b}{\left(1+y_{i}\right)^{b+1}}+2 \theta y_{i} \geq 0, \quad 1 \leq i \leq n, \\
& \sum_{i=1}^{n} y_{i}\left(\frac{-b}{\left(1+y_{i}\right)^{b+1}}+2 \theta y_{i}\right)=0 .
\end{aligned}
$$

Since $\boldsymbol{y} \geq \mathbf{0}$, we need to determine whether the constraint is binding or not. Namely, we need to determine whether or not there exist any components of $y$ that are zero. To do this, we compare the values of $f$ in all possible cases. Let $y$ has $k$ zero components, i.e., $k:=\left|\left\{i: y_{i}=0,1 \leq i \leq n\right\}\right|$. So, $0 \leq k \leq n$. If $0 \leq k<n$ then from (L2.7) $\theta>0$, from (L2.5) $g(y)=0$, and from (L2.8) all non-zero components of $\boldsymbol{y}$ are equal to each other. Thus, since $g(y)=0$, all non-zero components of $\boldsymbol{y}$ are equal to $[C /(n-k)]^{1 / 2}$. If $k=n$ then all components of $\boldsymbol{y}$ are zero and $f(\boldsymbol{y})=n$. Hence,

$$
f(y)=\left\{\begin{array}{ll}
k+\frac{n-k}{\left(1+\sqrt{\frac{G}{n-k}}\right)^{\prime \prime}} & 0 \leq k<n \\
n & k=n
\end{array} .\right.
$$

Next, we show that the expression in (L2.9) is minimized for $k=0$ and therefore, $y$ has no-zero components. To show this, we prove the validity of the following inequality: 


$$
x+\frac{n-x}{\left(1+\sqrt{\frac{c}{n-.1}}\right)^{b}} \geq \frac{n}{\left(1+\sqrt{\frac{c}{n}}\right)^{b}}, \quad x \in[0, n) .
$$

Define the function on the left-hand side of (L2.10) as $h(x)$. Taking the partial derivative of $h(x)$ with respect to $x$ and using Bernoulli's Inequality from [19], we obtain,

$$
\frac{\partial h}{\partial x}=1-\frac{1+\left(\frac{b}{2}+1\right) \sqrt{\frac{C}{n-1}}}{\left(1+\sqrt{\frac{C}{n-x}}\right)^{b+1}}>1-\frac{1+\left(\frac{b}{2}+1\right) \sqrt{\frac{C}{n-x}}}{1+(b+1) \sqrt{\frac{C}{n-x}}}=\frac{\frac{b}{2} \sqrt{\frac{C}{n-1}}}{1+(b+1) \sqrt{\frac{c}{n-x}}} \geq 0 .
$$

So, $h(x)$ is an increasing function of $x$ on $[0, n)$. Hence, $h(x) \geq h(0)$ for every $x \in[0, n)$. This completes the proof of (L2.10) and also implies that $f(y)$ assumes minimum value for $k=0$. This further implies that all components of $\boldsymbol{y}$ are equal to $(C / n)^{1 / 2}$ and that $f(y)=n /\left[1+(C / n)^{1 / 2}\right]^{b}$. Since $f(\boldsymbol{x}) \geq f(\boldsymbol{y})$ for every $\boldsymbol{x} \geq \mathbf{0},(\mathrm{L} 2.2)$ follows. $\square$

Next, in Lemma 2, we set $n=N_{t}, b=\gamma, x_{i}=u_{i m}(t)$, $C=m D^{2} / c_{2}$ for every $1 \leq i \leq n$, and $1 \leq m \leq n-1$, so that (L2.1) and (8) become identical. Also the left-hand side of (L2.2) and the inner summation in (9) become identical. Hence, we obtain the following lower bound on the lefthand side of (9):

$$
\sum_{m=1}^{N_{t}-1} \sum_{i=1}^{N_{t}} \frac{1}{\left(1+u_{i m}(t)\right)^{\gamma}} \geq \sum_{m=1}^{N_{t}-1} \frac{N_{t}}{\left(1+\frac{D}{\sqrt{c_{2}}} \sqrt{\frac{m}{N_{t}}}\right)^{\gamma}}
$$

Next, we define $d:=D / c_{2}{ }^{1 / 2}$. The quantity $d$ is the diameter of the network domain divided by a constant approximately equal to 0.625 . Combining this definition with (9) and (10), we obtain the following necessary condition for $N_{t}$ simultaneously successful transmissions at time $t$ :

$$
\begin{aligned}
& \sum_{m=1}^{N_{r}-1} \frac{1}{\left(1+d \sqrt{\frac{m}{N_{t}}}\right)^{\gamma}} \leq \frac{G}{\beta} \\
& \stackrel{(a)}{\Rightarrow} \int_{i}^{N_{t}}\left(1+d \sqrt{\frac{x}{N_{t}}}\right)^{-\gamma} d x \leq \frac{G}{\beta} \\
& \stackrel{\text { (b) }}{\Leftrightarrow} \frac{2 N_{i}}{d^{2}} \int_{1+\frac{d}{\sqrt{n_{t}}}}^{1+d} \frac{u-1}{u^{\gamma}} d u \leq \frac{G}{\beta},
\end{aligned}
$$

where step (a) follows from the fact that $\int_{a}^{b+1} f(x) d x \leq \sum_{m=a}^{b} f(m)$, whenever $a$ and $b$ are integers and $f(x)$ is a continuous and non-increasing function of $x$ on $[a, b+1]$, and step $(b)$ follows from changing the variable of the integration by defining $u=1+d\left(x / N_{t}\right)^{1 / 2}$. Next, we define the communication density at time $t, \sigma$, as follows:

$$
\sigma:=\sqrt{N_{1}} / d
$$

The quantity $\sigma^{2}$ is proportional to the average number of successful transmissions per unit area. Combining (11) and (12), the necessary condition for $N_{t}$ simultaneously successful transmissions at time $t$ becomes:

$$
2 \sigma^{2} \int_{1+\frac{1}{\sigma}}^{1+d}\left(u^{1-\gamma}-u^{-\gamma}\right) d u \leq \frac{G}{\beta}
$$

Next, we use the following lemma to obtain a closed form solution for the upper bound on $N_{t}$ :

Lemma 3: Let $a, x$, and $y$ be real numbers such that $x y \geq 1 \geq a$. Also, let $I:=2 x^{2} \int_{1+1 / x}^{1+y}\left(u^{a}-u^{a-1}\right) d u$. Then,
$I \geq \frac{2 x^{2}\left[a(1+y)^{a+1}-(a+1)(1+y)^{a}\right]}{a(a+1)}+\frac{2 x^{2}}{a(a+1)}-1, \quad a \leq 1$ and $a \notin\{-1,0\}$

$I \geq 2 x^{2} y-2 x^{2} \log (1+y)-1$, $a=0 \quad(\mathrm{~L} 3.2)$

$$
I \geq 2 x^{2} \log (1+y)-\frac{2 x^{2} y}{1+y}-1, \quad a=-1 \text {. (L3.3) }
$$

\section{Proof of Lemma 3:}

Firstly, consider the case when $a \leq 1$ and $a \notin\{-1,0\}$. Define

$$
\begin{aligned}
& R_{1}(x, a):=\frac{2 x^{2}}{2 x(x-a)\left(1+\frac{1}{x}\right)^{a}+a(a+1)}, a \in(-\infty,-1) \cup(0,1] \\
& R_{2}(x, a):=\frac{2 x(x-a)\left(1+\frac{1}{3}\right)^{n}}{2 x^{2}-a(a+1)}, \quad a \in(-1,0) .
\end{aligned}
$$

$R_{1}$ and $R_{2}$ are differentiable functions of $x$ and partial derivatives of them with respect to $x$ are

$$
\begin{aligned}
& \frac{\partial R_{1}}{\partial x}=\frac{\left.4 a(a+1) x^{2 a+1}[1+x)^{1-a}-x^{1-a}\right]}{(1+x)^{1-a}\left[2 x^{2}(1+x)^{a}-2 a x(1+x)^{a}+a(a+1) \cdot x^{a^{-2}}\right.} \geq 0, a \in(-\infty,-1) \cup(0,1] \\
& \frac{\partial R_{2}}{\partial x}=\frac{-2 a\left(1-a^{2}\right)(2 x-a)}{(1+x)^{1-a} \cdot x^{a}\left[2 x^{2}-a(a+1)\right]^{2}} \geq 0, \quad a \in(-1,0) .
\end{aligned}
$$

Also, $\lim _{1 \downarrow 0} R_{1}=\lim _{x \downarrow 0} R_{2}=0$. So, $R_{1}$ and $R_{2}$ are non-decreasing and non-negative functions of $x$. Finally, $\lim R_{1}=\lim _{1 \rightarrow \infty} R_{2}=1$. Therefore,

$$
\begin{aligned}
& R_{1}=\frac{2 x^{2}}{2 x(x-a)\left(1+\frac{1}{x}\right)^{a}+a(a+1)} \leq 1, a \in(-\infty,-1) \cup(0,1] \\
\Rightarrow & \frac{2 x(x-a)\left(1+\frac{1}{1}\right)^{a}}{a(a+1)} \geq \frac{2 x^{2}}{a(a+1)}-1, \quad a \in(-x,-1) \cup(0,1] \\
(\mathrm{L} 3.4) &
\end{aligned}
$$

and,

$$
\begin{aligned}
& R_{2}=\frac{2 x(x-a)\left(1+\frac{1}{x}\right)^{a}}{2 x^{2}-a(a+1)} \leq 1, \quad a \in(-1,0) \\
\Rightarrow & \frac{2 x(x-a)\left(1+\frac{1}{x}\right)^{a}}{a(a+1)} \geq \frac{2 x^{2}}{a(a+1)}-1,
\end{aligned}
$$

(L3.5)

Combining (L3.4) and (L3.5) we obtain

$$
\begin{aligned}
& \frac{2 x^{2}}{a(a+1)}-1 \leq \frac{2 x(x-a)\left(1+\frac{1}{x}\right)^{a}}{a(a+1)}, \quad a \leq 1 \text { and } a \notin\{-1,0\} \\
\Rightarrow & \frac{\left.2 x^{2} a(1+y)^{a+1}-(a+1)(1+y)^{a}\right]}{a(a+1)}+\frac{2 x^{2}}{a(a+1)}-1 \\
& \leq \frac{2 x^{2}\left[a(1+y)^{a+1}-(a+1)(1+y)^{a}\right]}{a(a+1)}+\frac{2 x(x-a)\left(1+\frac{1}{x}\right)^{a}}{a(a+1)}, a \leq 1 \text { and } a \notin\{-1,0\} \\
= & 2 x^{2}\left\{\frac{1}{(a+1)}\left[(1+y)^{a+1}-\left(1+\frac{1}{x}\right)^{a+1}\right]-\frac{1}{a}\left[(1+y)^{a}-\left(1+\frac{1}{x}\right)^{a}\right]\right\} \\
= & I, \quad a \leq 1 \text { and } a \notin\{-1,0\} .
\end{aligned}
$$

This completes the proof of (L3.1). We prove $(\mathrm{L} 3.2)$ and (L3.3) as follows: Let $i \in\{-1,0\}$. Then 


$$
\begin{aligned}
& \left.I\right|_{a=i} \stackrel{(a)}{=} \lim _{a \uparrow_{i}} I \\
& \quad \stackrel{(b)}{\geq} \lim _{a \uparrow_{i}}\left\{\frac{2 x^{2}\left[a(1+y)^{a+1}-(a+1)(1+y)^{a}\right]}{a(a+1)}+\frac{2 x^{2}}{a(a+1)}-1\right\} \\
& \quad \text { (c) } \begin{cases}2 x^{2} y-2 x^{2} \log (1+y)-1 & i=0, \\
2 x^{2} \log (1+y)-\frac{2 x^{2} y}{1+y}-1 & i=-1,\end{cases}
\end{aligned}
$$

where step ( $a$ ) follows from the continuity of $I$ at $a=i$, step (b) follows from (L3.1), and step (c) follows from L'Hôpital's Rule from [20]. This completes the proof of Lemma 3.

By setting $a=1-\gamma, x=\sigma$ and $y=d$ in Lemma 3, $I$ becomes equal to the left-hand side of (13). Hence, using (L3.1), (L3.2), and (L3.3), we obtain the following necessary conditions for $N_{t}$ simultaneously successful transmissions at time $t$ :

$$
\begin{array}{r}
\frac{2 \sigma^{2}\left(\frac{1-\gamma}{\left(1+d \gamma^{\gamma-2}\right.}-\frac{2-\gamma}{(1+d)^{\gamma-1}}\right)}{(1-\gamma)(2-\gamma)}+\frac{2 \sigma^{2}}{(1-\gamma)(2-\gamma)}-1 \leq \frac{G}{\beta}, \gamma \notin\{1,2\} \\
2 \sigma^{2} d-2 \sigma^{2} \log (1+d)-1 \leq \frac{G}{\beta}, \quad \gamma=1
\end{array}
$$

$$
2 \sigma^{2} \log (1+d)-\frac{2 \sigma^{2} d}{1+d}-1 \leq \frac{G}{\beta}, \quad \gamma=2
$$

Solving (14), (15), and (16) for $\sigma$ and substituting $\sigma$ from (12), we obtain the following upper bound on $N_{t}$ :

$$
N_{t} \leq U_{\gamma}
$$

where $U_{\gamma}$ is defined as in (T1.3), (T1.4) and (T1.5).

Recall that $N_{t}^{Q}$ is the maximum value of $N_{t}$ over all the placements of the nodes, the choice of the transmitters, their intended receivers, and the transmission powers, given that there are no restrictions on the value of $N$. Since there have been no restrictions on any of these parameters during the derivation of (17), hence, the right-hand side of (17) is also an upper bound on $N_{t}^{Q}$, which is not less than $N_{t}^{\max }$. This completes the proof of (T1.1) in Theorem 1 .

Finally, we prove (T1.2) and part (ii) of Theorem 1 as follows: Suppose there is a single receiver node and $N_{t}$ transmissions intended for this node at time $t$. Then, in (7), $l_{i j}(t)$ is equal to zero for every $i, j$, and $t$. Thus, $N_{t}$ will be no more than $1+G / \beta$. This shows that none of the nodes can receive more than $1+G / \beta$ simultaneously successful transmissions intended for itself. This completes the proof of part (ii) of Theorem 1. Now, (T1.2) follows from part (ii) and the fact that the total number of nodes in the network is equal to $N_{\text {total }}$. \\ upper bounds on per-SENSOR end-to-end throughput capacity}

In this subsection, firstly, we prove the following theorem:

Theorem 2: $\lambda_{e}$ and $\lambda_{n}$ have the following upper bounds:

$$
\begin{aligned}
& \lambda_{c} \leq \lambda_{m} \leq \frac{W_{\max } U_{\gamma}}{\bar{H} N}, \\
& \lambda_{c} \leq \lambda_{m} \leq \frac{W_{\max } M}{N}\left(1+\frac{G}{\beta}\right), \\
& \lambda_{c} \leq \lambda_{m} \leq \frac{W_{\max } N_{\text {total }}}{\bar{H} N}\left(1+\frac{G}{\beta}\right) .
\end{aligned}
$$

\section{Proof of Theorem 2:}

Let $t$ be an arbitrary time instant, and define the total information transmission rate of the network at time $t, C(t)$, as follows:

$$
C(t):=\sum_{i=1}^{N_{t}} W_{i}(t)
$$

where $W_{i}(t)$ is the transmission rate of the $i^{\text {th }}$ successful transmission at time $t$. By the definition of $\bar{H}$ in section $2-4$, each bit of information delivered to its destination is transmitted in $\bar{H}$ hops on the average. Therefore, the time average of $C(t)$ over the time interval $[0, \infty)$ is not less than $\bar{H} \Sigma_{i=1}^{N} \lambda_{i}=\bar{H} N \lambda$. Also, since $W_{i}(t) \leq W_{\max }$ and $N_{t} \leq N_{t}^{\max }$, we find that $C(t) \leq W_{\max } N_{t}^{\max }$. Thus, average of $C(t)$ over any time interval cannot exceed $W_{\max }$ multiplied by a time invariant upper bound on $N_{t}^{\max }$. Since the quantity $U_{\gamma}$ in (T1.1) is such an upper bound, this implies

$$
\bar{H} N \lambda \leq W_{\max } U_{\gamma} \text {. }
$$

On the other hand, due to part (ii) of Theorem 1, we know that no destination node can receive more than $1+G / \beta$ transmissions successfully. Since each transmission cannot occur at a rate more than $W_{\max }$, this implies that at the given time $t$, the total rate at which information is being delivered to the destinations cannot exceed $M W_{\max }(1+G / \beta)$. Hence, the total average rate at which information is delivered to the destinations over the time interval $[0, \infty)$, which is equal to $N \lambda$, cannot exceed $M W_{\max }(1+G / \beta)$. This implies

$$
N \lambda \leq M W_{\max }(1+G / \beta) \text {. }
$$

Hence, (18) and (19) imply that

$$
\begin{aligned}
& \lambda \leq \frac{W_{\max } U_{y}}{\bar{H} N}, \\
& \lambda \leq \frac{W_{\max } M}{N}\left(1+\frac{G}{\beta}\right) .
\end{aligned}
$$

It is worth emphasizing that, because of the generality of the network model underlying the derivation of (20) and (21), they are applicable even when the mobility pattern of the nodes, the spatial-temporal transmission scheduling policy, the temporal variation of transmission powers, the sensor-destination pairs, and the possibly multi-path routes between them are optimally chosen as to maximize $\lambda$. Similarly, (20) and (21) are applicable even when the nodes are capable of maintaining multiple transmissions and/or receptions simultaneously.

Recall that $\lambda_{m}$ is the supremum of all end-to-end throughputs $\lambda_{0}$ for which there exist: a mobility pattern of the nodes, a traffic pattern, a spatial-temporal transmission scheduling policy, and a temporal variation of transmission powers, so that $\lambda \geq \lambda_{0}$. There have been no restrictions on these parameters during the derivation of (20) and (21). Hence, the right-hand sides of (20) and (21) are also upper 
bounds on $\lambda_{m}$, which is not less than $\lambda_{e}$. This completes the proof of (T2.1) and (T2.2). The proof of (T2.3) is identical with the proof of (T2.1) except that the expression on the right-hand side of (T1.2) is used instead of $U_{\gamma}$.

So far, there have been no restrictions on the number of simultaneous transmissions and/or receptions that a node is capable of maintaining. If, as in [1]-[4], there is also the additional restriction that a node cannot transmit and receive simultaneously and that a node is capable of maintaining at most one transmission or one reception at any given time, then the upper bounds on $\lambda_{e}$ and $\lambda_{n}$ can be further tightened. We will henceforth refer to this case as the half-duplex restricted case. In this case, no destination node can receive more than a single transmission, and thus following the same derivation method that was used between (18) and (19) shows that $\lambda \leq W_{\max } M / N$. Combining this inequality with (18), and following the same derivation method that was used after (19) in the proof of Theorem 1 lead to the following upper bound on $\lambda_{c}$ and $\lambda_{m}$ for the halfduplex restricted case:

$$
\lambda_{e} \leq \lambda_{m} \leq \frac{W_{\max }}{N} \min \left\{\frac{U_{\gamma}}{\bar{H}}, M\right\} .
$$

Finally, we show that dividing the communication bandwidth into several sub-channels of smaller bandwidth does not change the terms other than $W_{\max }$ in all of the results on $\lambda_{c}$ and $\lambda_{m}$ that we have presented so far. An assumption behind those results is that all transmissions are taking place in the same communication bandwidth. If the communication bandwidth is partitioned into several subchannels of smaller bandwidth, then there still is an upper bound on the transmission rate in each of these subchannels. All of the upper bounds on simultaneous transmission capacities of $\boldsymbol{Q}$ and the network are still valid for each of these sub-channels individually. Therefore, if there are $K$ sub-channels and the transmission rate of the $k^{\text {th }}$ sub-channel is no more than $W_{k}^{\max }$, then all of the upper bounds on $\lambda_{c}$ and $\lambda_{m}$ are still valid if $W_{\max }$ is replaced with $\sum_{h=1}^{k} W_{k}^{\text {max }}$.

\section{ANALYSIS OF THE UPPER BOUNDS}

In this section, we analyze how the upper bounds on $N_{1}^{Q}$, $N_{t}^{\text {max }}, \lambda_{c}$, and $\lambda_{n}$ are affected as various parameters of the network are varied individually.

Firstly, we analyze the asymptotic and limiting behavior of the upper bound $U_{\gamma}$ in Theorem 1, to draw the following conclusions about $N_{t}^{Q}$ :

$$
\begin{aligned}
& \text { - } \lim _{\gamma \rightarrow \infty} \frac{c_{i}}{\dot{r}^{2}}=\frac{1}{2 c_{2}}\left(1+\frac{G}{\beta}\right) D^{2} \quad \Rightarrow N_{t}^{Q} \text { is } O\left(\gamma^{2}\right) \\
& \text { - } \lim _{D \rightarrow \infty} \frac{\left.U_{i}\right|_{y=1}}{D}=\frac{1}{2 c_{2}{ }^{1 / 2}}\left(1+\frac{G}{\beta}\right) \quad \Rightarrow N_{t}^{Q} \text { is } O(D) \text { if } \gamma=1 \\
& \text { - } \lim _{\substack{D \rightarrow \infty \\
(\gamma<2)}} \frac{l^{\prime}}{D^{\gamma}}=\frac{1}{c_{2}^{\gamma / 2}}\left(1-\frac{\gamma}{2}\right)\left(1+\frac{G}{\beta}\right) \Rightarrow N_{t}^{Q} \text { is } O\left(D^{\gamma}\right) \text { if } \gamma<2 \\
& \text { - } \lim _{D \rightarrow \infty} \frac{\left.U_{i}\right|_{\operatorname{loz}}}{D^{2} / \log (D)}=\frac{1}{2 c_{2}}\left(1+\frac{G}{\beta}\right) \Rightarrow N_{t}^{Q} \quad \text { is } O\left(D^{2} / \log (D)\right) \text { if } \\
& \gamma=2
\end{aligned}
$$

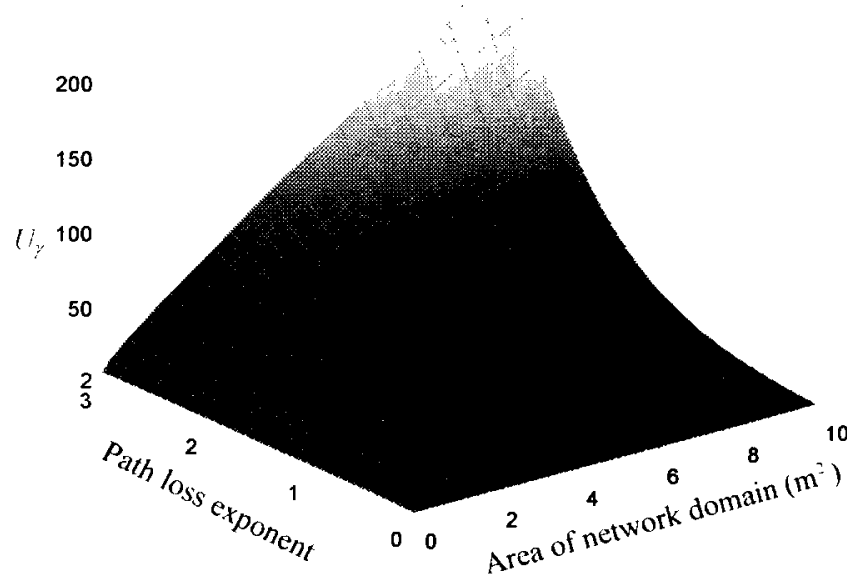

Figure 3 - Upper bound on the simultaneous transmission capacity of the network domain as a function of the area of the network domain and the path loss exponent

- $\lim _{\substack{D \rightarrow \infty \\(\gamma>2)}} \frac{U_{\gamma}}{D^{2}}=\frac{(\gamma-1)(\gamma-2)}{2 c_{2}}\left(1+\frac{G}{\beta}\right) \quad \Rightarrow N_{i}^{Q}$ is $O\left(D^{2}\right)$ if $\gamma>2$

- $\lim _{G / \beta \rightarrow \infty} \frac{U_{\gamma}}{G / \beta}=f(\gamma, d):=\frac{U_{\gamma}}{1+G / \beta} \Rightarrow N_{t}^{Q}$ is $O(G / \beta)$

- $\lim _{\gamma \downarrow_{0}} U_{\gamma}=\lim _{D \downarrow_{0}} U_{\gamma}=1+\frac{G}{\beta} \Rightarrow \begin{gathered}\text { Lack of attenuation is } \\ \text { equivalent to lack of space }\end{gathered}$

Also, since the area of the network domain is $A=\pi D^{2} / 4$, $D$ can be replaced with $(4 A / \pi)^{1 / 2}$. Doing so, we can also conclude that $N_{t}^{Q}$ is $O\left(A^{\min \{\gamma / 2.1\}}\right)$ if $\gamma \neq 2$ and $O(A / \log (A))$ if $\gamma=2$. Regardless of the value of $\gamma$, this also implies that $N_{t}^{Q}$ cannot grow with the area of $\boldsymbol{Q}$ super-linearly. Linear growth is not possible when $\gamma \leq 2$, and can only be possible when $\gamma>2$.

In Figure 3, $U_{y}$ is plotted as a function of $A$ and $\gamma$, for $G=\beta=10$. This figure illustrates the growth trend of $U_{\gamma}$ as $\gamma$ and/or $A$ increase. It is possible to observe the linear and the sub-linear growth of $U_{\gamma}$ with $A$ when $\gamma>2$ and $0<\gamma \leq 2$, respectively. The figure also illustrates the equivalence of the lack of attenuation $(\gamma=0)$ and the lack of space $(A=0)$. One should also notice the quadratic growth of $U_{\gamma}$ with $\gamma$.

Secondly, we analyze the upper bounds on $N_{t}^{\max }$. Inequality (T1.1) of Theorem 1 shows that $N_{t}^{\max }$ is $O(1)$ with respect to $N$. Since $N_{t}^{\max } \leq N_{t}^{Q}$, all of the above asymptotic results are valid for $N_{t}^{\max }$, too.

However, from (T1.2) and the facts that $N_{\text {toral }} \leq N+M$ and $M \leq N$, we find that $N_{t}^{\max } \leq 2 N(1+G / \beta)$. Therefore, for a given $N, G$, and $\beta$, the upper bound on $N_{t}^{\max }$ in (T1.1) loses its tightness beyond some finite values of $D$ and $\gamma$. Existence of an upper bound on $N_{t}^{\max }$ independent of $D$ and $\gamma$ also shows that $N_{t}^{\max }$ is $O(1)$ with respect to $A$ and $\gamma$. The reason is that beyond some finite values of $A$ or $\gamma$, the network domain provides sufficient space and attenuation, so that the upper bound on the number of simultaneous receptions per-node, i.e., $1+G / \beta$, becomes the limiting factor.

Next, we analyze asymptotic and limiting behavior of the upper bounds on $\lambda_{e}$ and $\lambda_{m}$. Inequality (T2.1) of Theorem 2 shows that $\lambda_{e}$ and $\lambda_{m}$ are $O(1 / N)$ and $O(1 / \bar{H})$. It also shows 


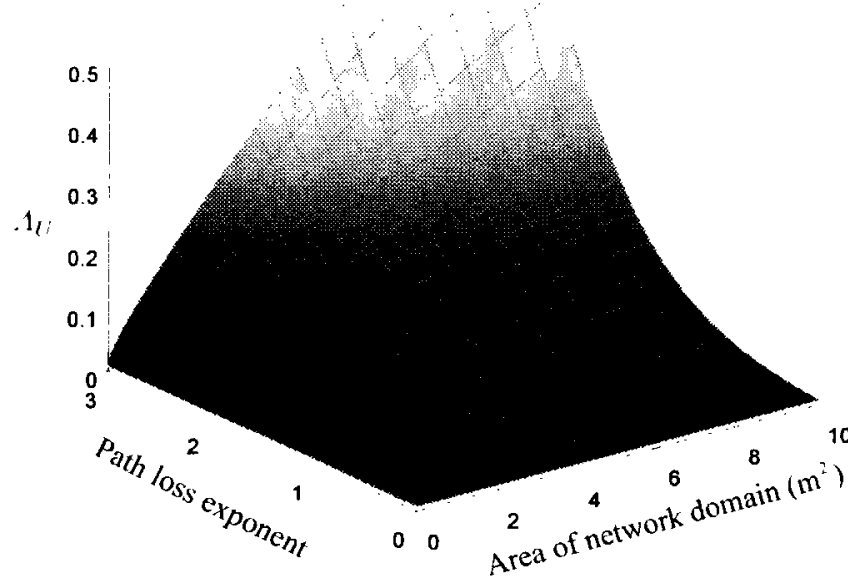

Fig. 4 - Upper bound on the normalized per sensor end-to-end throughput capacity as a function of the area of the network domain and the path loss exponent $n=$

that $\lambda_{c}$ and $\lambda_{m}$ are $O(G / \beta) .{ }^{9}$ We also observe that $\lambda_{c}$ and $\lambda_{m}$ are upper bounded by $W_{\max }(1+G / \beta) /(\bar{H} N)$ when the network domain lacks attenuation or space. Due to (T2.2), $\lambda_{c}$ and $\lambda_{m}$ cannot exceed $W_{\max } M(1+G / \beta) / N$, which is independent of $A$ and $\gamma$. So, the upper bound in (T2.2) becomes more restrictive than the upper bound in (T2.1) beyond some finite values of $A$ or $\gamma$, and thus $\lambda_{e}$ and $\lambda_{m}$ are $O(1)$ with respect to $A$ and $\gamma$. Similar behavior is also observable in the half-duplex restricted case; for example, if the set of sensor nodes and the set of destination nodes are disjoint and $\bar{H}=1,{ }^{10}$ then beyond some finite values of $A$ or $\gamma$, the network domain provides sufficient space and attenuation so that at any given time, there is a placement of the nodes for which $M$ simultaneously successful transmissions can be established between $M$ of the $N$ sensors and the $M$ destinations. However, no more transmissions can be scheduled, since there are no remaining inactive destination nodes, and thus $\lambda_{e}$ and $\lambda_{m}$ cannot exceed $W_{\max } M / N$, which can be observed from (22). In general, there is a region of $(A, \gamma)$ pairs for which the dominant upper bound on $\lambda_{e}$ and $\lambda_{m}$ is $W_{\max } U_{\gamma} /(\bar{H} N)$. From (T2.1) and (T2.2), it can observed that this region is contained within the region bounded by the $A$ axis, the $\gamma$ axis, and the set of $(A, \gamma)$ pairs for which $U_{\gamma}=M \bar{H}(1+G / \beta)$. Since $U_{\gamma}$ is an increasing function of $A$ and $\gamma$, this region will expand as $M$ (and thus $N$, since $M \leq N$ ) increases. This shows that the limitation of $\lambda_{e}$ and $\lambda_{m}$ due to shortage of space and attenuation is more pronounced when $M$ (and thus $N$ ) is large compared to $U_{\gamma}$. Additionally, we have shown that $U_{\gamma}$ is $\Theta\left(A^{\min \{\gamma / 2,1\}}\right)$ when $\gamma \neq 2, \Theta(A / \log (A))$ when $\gamma=2$, and also $\Theta\left(\gamma^{2}\right)$. These observations support the claim that for large $M$ (and thus $N$ ) there is a region of $(A, \gamma)$ pairs where additional space and

${ }^{9}$ The $O(G / \beta)$ result assumes that $W_{\max }$ is not dependent on $G / \beta$. However, in some practical systems, $W_{\max }$ is inversely proportional to $G / \beta$, as we will see in section 7 .

${ }^{10}$ Note that this is the least possible value of $\bar{H}$, and is achieved when each sensor node transmits the information that it generates directly to its destination node.

91

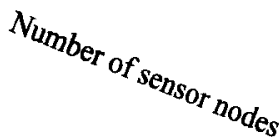

Fig. 5 - Upper bound on the normalized per sensor end-toend

throughput capacity as a function of the area of the

additional attenuation provide considerable increase in $\lambda_{e}$ and $\lambda_{m}$, where the behavior of $\lambda_{e}$ and $\lambda_{m}$ resembles the asymptotic behavior of $U_{\gamma}$, and beyond this region the behavior $\lambda_{e}$ and $\lambda_{m}$ changes into $\Theta(1)$ with respect to $A$ and $\gamma$

Next, we demonstrate the above results through an example. Consider the half-duplex restricted case. For this case, we have shown that $\lambda_{e}$ and $\lambda_{m}$ cannot exceed the righthand side of (22). Now, we normalize this quantity with respect to $W_{\max }$, and we denote the resulting expression by $\Lambda_{U}$. In Figure $4, \Lambda_{U}$ is plotted as a function of $A$ and $\gamma$. The other parameters for this example are: $G=\beta=10, N=250$, $M=N / 2$, and $\bar{H}=1$. This figure illustrates the variation in the growth trend of $A_{U}$ as a function of $A$ for various values of $\gamma$. Also, it demonstrates the presence of a region of $(A, \gamma)$ pairs where the limitation of $\lambda_{c}$ and $\lambda_{m}$ is due to shortage of space and attenuation. For the $(A, \gamma)$ pairs outside of this region, shortage of inactive destination nodes becomes the dominant limitation, and thus $W_{\max } M / N$ becomes the dominant upper bound on $\lambda_{e}$ and $\lambda_{n}$ (this can also be observed from (22)).

In Figure 5, parameter values are the same except that $N$ is now an independent variable (so is $M$, since $M=N / 2$ in this example) and $\gamma=3$. The light green region consists of the $(A, N)$ pairs where the limitation of $\lambda_{c}$ and $\lambda_{n}$ is due to shortage of space. For the $(A, N)$ pairs outside of this region, namely inside the darker blue region, shortage of inactive destination nodes is the dominant limitation, and thus $W_{\max } M / N$ is the dominant upper bound on $\lambda_{e}$ and $\lambda_{m}$. The figure also demonstrates that if the area of the network domain is kept constant and the number of sensor nodes is increased, then $\Lambda_{U}$ decays as $\Theta(1 / N)$, so that $\lambda_{e}$ and $\lambda_{m}$ vanish as $N$ grows large. However, if the area also increases with $N$, we observe that it can be possible to keep $\Lambda_{U}$ at a constant level so that it does not rule out the possibility of achieving a non-vanishing per-sensor end-to-end throughput as the number of sensor nodes grows large. We will elaborate on this result in section 7 . 


\section{$\lambda_{M}$ AND $\lambda_{M}$ ARE $\theta(1 / N)$}

In the previous section, we have shown that $\lambda_{c}$ and $\lambda_{m}$ are $O(1 / N)$. Next, to prove that they are also $\Theta(1 / N)$, we will show that they are $\Omega(1 / N)$. We do this by constructing a TDMA scheme that assigns each of the sensor nodes a separate time slot of constant duration. In such a scheme, there are $N$ slots in each cycle and each of the sensor nodes transmits directly to its destination in the slot assigned to itself, with a transmission power large enough to satisfy the signal to noise ratio requirement. Assuming $\zeta$ is an upper bound on the power of noise in the used communication bandwidth, a transmission power of $\beta \zeta / a(D)$ guarantees successful reception.

Although this simple scheme takes no advantage of spatial reuse, it allows each of the sensor nodes transmitting $1 / N$ fraction of the time. Thus, assuming that each transmission satisfying the signal-to-noise ratio requirement can occur with rate $W$, an node end-to-end throughput of $W / N$ is achievable by all sensor nodes. This shows that $\lambda_{e}$ and $\lambda_{m}$ are $\Omega(1 / N)$. As a result, $\lambda_{e}$ and $\lambda_{m}$ are $\Theta(1 / N)$.

\section{IMPLICATIONS OF THE RESULTS ON SCALABILITY}

In this section, we consider the following scalability problem: we are increasing the number of sensor nodes in the network indefinitely, and we want to achieve a desired per-sensor end-to-end throughput, say $\lambda_{0}$. $\lambda_{0}$ is not achievable if no other parameter is increased as a function of $N$, since due to (T2.1) $\lambda_{e}$ and $\lambda_{m}$ are no more than $W_{\text {max }} U_{y} /(\bar{H} N)$, which is $O(1 / N)$. So, one or more of the parameters from $W_{\text {nax }}, \gamma, G / \beta$, or $A$ must increase with $N$ and $N$ must be increasing according to a function of $U_{\gamma}$, so that $W_{\max } U_{\gamma} /(\bar{H} N) \geq \lambda_{0}$ (note that $\bar{H}$ cannot be indefinitely reduced to compensate for increasing $N$, because $\bar{H} \geq 1$, as every bit of information has to be transmitted for at least one hop). This shows that $\bar{H} N$ must be $O\left(W_{\max } U_{\gamma}\right)$.

For practical systems, $\gamma$ is a property of the wireless channel and it cannot increase with $N$. $W_{\max }$ cannot increase indefinitely with $N$, because of the presence of noise and because of the maximum transmission power constraints. These limit reliable information transmission to rates that do not grow with $N$. On the other hand, $G / \beta$ depends on the implementation of the communication system and increasing it for a given system bandwidth usually requires decreasing $W_{\max }$. For example, it is shown in [13] that in spread spectrum CDMA, for a given system bandwidth, symbol transmission rate is inversely proportional to the processing gain. Likewise, reducing $\beta$ requires a proportional decrease in the symbol transmission rate to satisfy a given bit error rate requirement. Therefore, increasing $G / \beta$ will not compensate for increasing $N$. So, the only way of achieving $\lambda_{0}$ would be increasing $A$ as $N$ increases. Hence, $N$ must be increasing as a function of $A$. We have shown that $U_{\gamma}$ is $\Theta\left(A^{\min \{\gamma / 2.1\}}\right)$ when $\gamma \neq 2$ and $\Theta(A / \log (A))$ when $\gamma=2$. Therefore, unless $N$ is $O\left(A^{\min \{\gamma / 2.1\}}\right)$ when $\gamma \neq 2$ and $O(A / \log (A))$ when $\gamma=2, \lambda_{0}$ is not achievable. Also, $\bar{H}$ must be $\Theta(1)$ with respect to $N$ due to the following reasoning. We know that $\bar{H} \geq 1$, which implies that $\bar{H}$ is $\Omega(1)$. To see why $\bar{H}$ must be $O(1)$ with respect to $N$, observe that $\lambda_{0}$ cannot exceed $2 W_{\max }(1+G / \beta)$ $/ \bar{H}$ due to (T2.3) and the fact that $M \leq N$. Since increasing $G / \beta$ requires a proportional reduction in $W_{\max }$, as is the case in spread spectrum CDMA, we find that compensating for indefinitely growing $\bar{H}$ by increasing $G / \beta$ is not possible.

On the other hand, $\lambda_{0}$ is not achievable unless the upper bound in (T2.2) is at least as big as $\lambda_{0}$. Hence, (T2.2) implies that if $M$ is not $\Omega(N)$, then $\lambda_{0}$ is not achievable, because the above argument in the previous paragraph shows that $W_{\max }(1+G / \beta)$ cannot grow indefinitely with $N$.

The above results can also be stated in terms of the sensor density, $\rho:=N / A$. From the above $O(\cdot)$ results, dividing $N$ and the asymptotic upper bounds on $N$ by $A$, we obtain the following result: unless $\rho$ is $O\left(A^{\min \{\gamma / 2-1,0\}}\right)$ when $\gamma \neq 2$ and $O(1 / \log (A))$ when $\gamma=2, \lambda_{0}$ is not achievable. In other words, $\lambda_{0}$ is not achievable if $\rho$ grows with $N$ indefinitely when $\gamma>2$, if $\rho \log (A)$ grows with $N$ indefinitely when $\gamma=2$, and if $\rho A^{1-\gamma / 2}$ grows with $N$ indefinitely when $\gamma<2$. In any case, $\lambda_{0}$ is not achievable if $\rho$ grows with $N$ indefinitely. Also, when $\gamma \leq 2$, unless $\rho$ decays down to 0 as $N \rightarrow \infty, \lambda_{0}$ is not achievable. Our observations in this and the previous two paragraphs prove the following corollary regarding practical systems:

Corollary: (A necessary condition for the scalability of practical systems) $A$ desired per-sensor end-to-end throughput is not achievable as $N \rightarrow \infty$, unless $\bar{H}$ is $\Theta(1)$ with respect to $N, M$ and $A$ grow with $N$, such that $M$ is $\Omega(N)$, and the following equivalent conditions are satisfied: - $N$ is $O\left(A^{\min \{\gamma / 2.1\}}\right)$ when $\gamma \neq 2$ and $O(A / \log (A))$ when $\gamma=2$, - $\rho$ is $O\left(A^{\min \{\gamma / 2-1.0\}}\right)$ when $\gamma \neq 2$ and $O(1 / \log (A))$ when $\gamma=2$.

Figure 6 illustrates this corollary. In this figure, $G=\beta=10, \bar{H}=1, M=N / 2$ and the curves are obtained by plotting the $(A, N)$ pairs, for which $A_{U}=0.1$ and $\gamma \in\{0,1,2,3\}$. We know that normalized $\lambda_{i}$ and $\lambda_{m}$ are no more than $\Lambda_{U}$. Also, it follows from the definition of $\Lambda_{U}$ that $\Lambda_{U}$ is a decreasing function of $N$ and an increasing function of $A$ when $\Lambda_{U}<M / N=0.5$. Therefore, each of these curves separates a region of $(A, N)$ pairs where a normalized end-to-end throughput of 0.1 is not achievable and another region where it may be achievable on average or by all sensor nodes. For example, when $\gamma=2$, and $(A, N)=(3,400)$, the normalized end-to-end throughput 0.1 is not achievable, whereas it may be achievable on average or by all sensor nodes for $(A, N)=(3,100)$. The corollary tells us that for the sequence of $(A, N)$ pairs forming each of the curves in Figure $6, N$ is $\Theta(1), \Theta\left(A^{1 / 2}\right)$, $\Theta(A / \log (A))$, and $\Theta(A)$ when $\gamma$ is $0,1,2$ and 3 , respectively. Equivalently, for the sequence of $(A, \rho)$ pairs associated with each of these curves, $\rho$ is $\Theta(1 / A), \Theta\left(1 / A^{1 / 2}\right), \Theta(1 / \log (A))$, and $\Theta(1)$ when $\gamma$ is $0,1,2$ and 3 , respectively.

\section{CONCLUSIONS}

In this paper, we have studied the capacity of single-userdetection based wireless sensor networks through the use of a more general network model than the models used in the literature. 
Instead of the propagation model used in the previous studies, we used the bounded power law decaying propagation model, which was proposed in other studies on connectivity such as [15] and [16], to obtain more realistic results for small transmitter-receiver distances, while approximating the conventional model at large distances. Using this model, we concluded that $N_{t}^{\max }$ cannot exceed $N_{l}^{Q}$, which ${ }^{11}$ is independent of $N$, but depends on $A, \gamma, G$, and $\beta$. The analysis of the upper bound on $N_{t}^{Q}$ in Theorem 1 has revealed that $N_{t}^{Q}$ is $O\left(A^{\min \{\gamma / 2.1\}}\right)$ for $\gamma \neq 2$ and is $O(A / \log (A))$ for $\gamma=2$. The analysis has also shown that $N_{t}^{Q}$ is $O\left(\gamma^{2}\right)$ and $O(G / \beta)$.

Additionally, since the network model that we have used is quite general, our results in this paper do not only hold for the network scenarios of [1]-[4], but also hold for networks whose nodes move with any mobility pattern or are capable of maintaining any number of simultaneous transmissions and/or receptions. Hence, we have been able to show that the maximum achievable per-sensor end-toend throughput is $\Theta(1 / N)$, even when the mobility pattern of the sensor and the destination nodes, the spatial-temporal transmission scheduling policy, the temporal variation of transmission powers, the sensor-destination pairs, and the possibly multi-path routes between the sensors and the destinations are optimally chosen. Furthermore, this result holds even the communication bandwidth is partitioned into sub-channels of smaller bandwidth.

Moreover, our results are valid for any nonnegative value of $\gamma^{12}$ This allowed us to characterize the behavior of $N_{t}^{Q}$, $N_{t}^{\max }, \lambda_{c}$, and $\lambda_{n}$ under low attenuation conditions. In particular, it allowed us to show that lack of attenuation and lack of space are equivalent, where $N_{t}^{\max }$ and $N_{t}^{Q}$ cannot exceed $1+G / \beta$. Also, in these equivalent cases, $\lambda_{e}$ and $\lambda_{n t}$ cannot exceed $W_{\max }(1+G / \beta) /(\bar{H} N)$.

We have also shown that no node can receive more than $1+G / \beta$ simultaneously successful transmissions intended for itself. This allowed us to show that $N_{t}^{\max }, \lambda_{e}$, and $\lambda_{n}$ are $O(1)$ with respect to $A$ and $\gamma$ for a given $N$. Together with (T2.1) and (T2.2), this also allowed us to justify that the limitation of $\lambda_{r}$ and $\lambda_{n}$ due to shortage of space and attenuation is more pronounced when $M$ and $N$ are large.

Finally, we have studied the implications of our results on the scalability of wireless sensor networks. We have shown that as $N$ becomes large, unless one or more of the parameters from $W_{\max }, \gamma, G / \beta$, or $A$ grows with $N$, and $\bar{H} N$ is $O\left(W_{\max } U_{\gamma}\right)$, a desired per-sensor end-to-end throughput is not achievable. Regarding scalability of practical systems, we have concluded that $M$ must be $\Omega(N)$ and $\bar{H}$ must be $\Theta(1)$ with respect to $N$. Moreover, we have concluded that $A$ is the only remaining parameter whose growth can compensate for increasing $N$. Above all, we have proved that as $N \rightarrow \infty$, a desired per-sensor end-to-end throughput is not achievable, unless $A$ also grows with $N$, and $N$ is $O\left(A^{\min (\gamma / 2,1\}}\right)$ when $\gamma \neq 2$ and is $O(A / \log (A))$ when $\gamma=2$.

\footnotetext{
"The difference between our results and the results in [1] and [2], which concluded that $N_{t}^{\max }$ is $\Theta(N)$, is due to the bounded nature of our propagation model.

${ }^{12}$ Note that the results of the related studies such as [1] and [2] are linited to values of $\gamma$ that exceed 2.
}

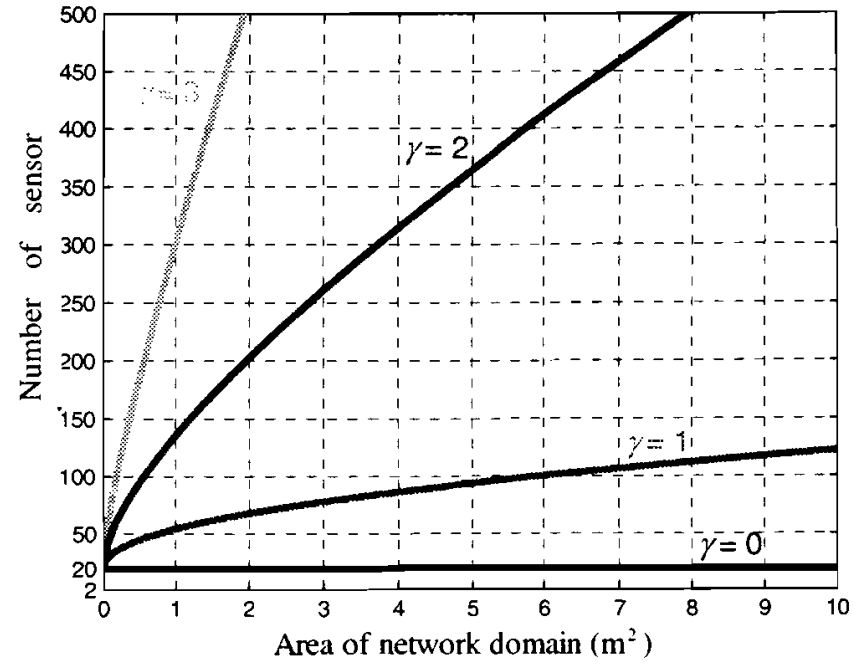

Figure 6 - Curves formed by the $(A, N)$ pairs for which $\Lambda_{l^{\prime}}=0.1$. For the $(A, N)$ pairs above the curves. any normalized throughput greater than or equal to 0.1 is not achievable

In summary, in this paper, we analyzed the capacity of single-user-detection based wireless sensor networks through the use of a more general network model, and we determined several necessary conditions for the scalability of such networks. This was performed by considering only one of the fundamental requirements for scalability, which is the requirement of a non-vanishing per-sensor end-to-end throughput as the number of sensor nodes grows large. An interesting extension of this work would be to determine the additional necessary conditions that result from other fundamental requirements for scalability, such as bounded end-to-end delay, bounded power consumption, bounded processing power, and bounded memory consumption at the nodes.

\section{REFERENCES}

[1] P. Gupta and P. R. Kumar, "The capacity of wireless networks," IEEE Transactions on Information Theory, vol. 46, no. 2, pp. 388-404, March 2000.

[2] M. Grossglauser and D. Tse. "Mobility increases the capacity of wireless adhoc networks," IEEE/ACM Trans. Networking, vol. 10, no. 4, pp. 477-486, August 2002.

[3] D. Marco, E. J. Duarte-Melo, M. Liu, D. L. Neuhoff, "On the Many-to-one Transport Capacity of a Dense Wireless Sensor Network and the Compressibility of Its Data," in IPSN 2003, pp. 1-16, Palo Alto, CA, April 2003.

[4] A. Chakrabarti, A. Sabharwal, B. Aazhang, "Multi-hop communication is Order-Optimal for Homogeneous Sensor Networks," in IPSN 2004, pp. 178-185, Berkeley, CA, April 2003.

[5] S. Toumpis and A. J. Goldsmith "Capacity regions for wireless ad hoc networks," IEEE Trans. Wireless Communications, vol. 2, no. 4, pp. 736-748, July 2003.

[6] J. Li, C. Blake, D. D. Couto, H. Lee and R. Morris, "Capacity of ad hoc wireless networks," in ACM Mobicom, Rome, Italy, July 2001.

[7] S. Yi, Y. Pei and S. Kalyanaraman, "On the capacity improvement of ad hoc wireless networks using directional antennas," in ACM MobiHoc, Annapolis, MD, June 2003.

[8] C. Peraki and S. D. Servetto, "On the maximum stable throughput problem in random networks with directional antennas," in Proceedings of ACM MobiHoc, Annapolis, MD, June 2003. 
[9] B. Liu, Z. Liu and D. Towsley. On the capacity of hybrid wireless networks. in IEEE Infocom, San Francisco, CA, April 2003.

[10] P. Gupta and P. R. Kumar, "Towards an information theory of large networks: an achievable rate region," IEEE Trans. Inform. Theory, vol. 49, no. 8, pp. 1877-1894, August 2003.

[11] M. Gasptar and M. Vetterli, "On the capacity of wireless networks: The relay case," in Proceedings of IEEE Infocom. New York, NY, June 2002.

[12] H. El Ganal, "On the scaling laws of dense wireless sensor networks," submitted to IEEE Trans. Inform. Theory, April 2003.

[13] T. S. Rappaport, Wireless Communications, Principles and Practice, NJ: Prentice Hall, 1996, pp. 104, 276-278.

[14] J. B. Andersen and T. S. Rappaport, S. Yoshida, "Propagation measurements and models for wireless communications channels," IEEE Communications Magazine, pp. 42-49, January 1995.

[15] F. Baccelli and B. Blaszczyszyn, "On a coverage range process ranging from the boolean model to the Poisson Voroni tesellation with applications to wireless communications." Adv'. Appl. Prob., vol. 33, no. 2, pp. 293323,2001 .

[16] O. Dousse, F. Baccelli, P. Thiran, "Impact of Interferences on Connectivity in Ad Hoc Networks," in Proceedings of IEEE Infocom, San Francisco, CA, April 2003.

[17] J. Pach, P. K. Agarwal, Combinatorial geometry, New York : Wiley, 1995, pp. 207-221.

[18] M. J. Panik, Classical optimization : foundations and extensions, American Elsevier Publishing Company, 1976. pp: 227-306.

[19] D. S. Mitrinovic, Analytic Inequalities, New York: SpringerVerlag, 1970, pp: 10-36.

W. Rudin, Principles of Mathematical Analysis, third edition. Mc Graw Hill,

Onur Arpacioglu received his B.Sc. in Electrical and Electronic Engineering degree in 2001 with the highest honor from Bogazici University, Istanbul, Turkey. He is currently working toward his Ph.D. degree in the School of Electrical and Computer Engineering, Cornell University, Ithaca, NY, USA. His research interests include scalability and capacity of wireless networks.

Zygmunt J. Haas received his B.Sc. in EE in 1979 and M.Sc. in EE in 1985. In 1988, he earned his Ph.D. from Stanford University and subsequently joined AT\&T Bell Laboratories in the Network Research Department. There he pursued research on wireless communications, mobility management, fast protocols, optical networks, and optical switching. From September 1994 till July 1995, Dr. Haas worked for the AT\&T Wireless Center of Excellence, where he investigated various aspects of wireless and mobile networking, concentrating on TCP/IP networks. As of August 1995, he joined the faculty of the School of Electrical and Computer Engineering at Cornell University. Dr. Haas is an author of numerous technical papers and holds fifteen patents in the fields of high-speed networking, wireless networks, and optical switching. He has organized several workshops, delivered numerous tutorials at major IEEE and ACM conferences, and serves as editor of several journals and magazines, including the IEEE Transactions on Networking, the IEEE Transactions on Wireless Communications, the IEEE Communications Magazine, and the ACM/Kluwer Wireless Networks journal. He has been a guest editor of IEEE JSAC issues on "Gigabit Networks," "Mobile Computing Networks," and "Ad-Hoc Networks". Dr. Haas is a Senior Member of IEEE, and the Chair of the IEEE Technical Committee on Personal Communications. His interests include: mobile and wireless communication and networks, personal communication service, and high-speed communication and protocols.

His e-mail is: haas@ece.cornell.edu and his URL is: http://wnl.ece.cornell.edu 